\title{
Content-Aware Power Saving Multimedia Adaptation for Mobile Learning
}

\author{
Syed Asim Jalal, Nicholas Gibbins, David Millard, Bashir Al-Hashimi, Naif Radi Aljohani \\ School of Electronics and Computer Science \\ University of Southampton \\ Southampton, United Kingdom \\ \{smaj08r,nmg,dem,bmah, nra1d10\}@ecs.soton.ac.uk
}

\begin{abstract}
Due to the tremendous enhancements in the capabilities of mobile devices in recent years and accessibility to higher bandwidth mobile internet, the use of online multimedia learning resources on mobile devices is increasingly becoming popular. Improvements in battery capacity have not matched the same advancements compared to other features of mobile devices. Limited Battery power is introducing a significant challenge in making better use of online educational multimedia resources. Online Multimedia Resources drains more battery power as a result of higher amount of wireless data transfer and therefore limiting learning opportunities on the move. Many power saving multimedia adaptation techniques have been suggested. Majority of these techniques achieve battery efficiency while reducing multimedia quality. So far, however, to the best of our knowledge no previous effort has considered the factor of learning efficacy in multimedia adaptation process. Existing adaptation techniques are susceptible to information loss as a result of quality of reduction. Such loss affects the learning content efficacy and jeopardizes the learning process. In this paper, we recommend a novel power save educational multimedia adaptation approach that considers the learning aspect of multimedia in the adaptation process. Our technique enables learning for extended duration by battery power saving without putting the learning process at risk. Efficacy of entire learning resources is managed by not allowing any part of the learning multimedia to be delivered in a quality that will negatively affect the learning outcome. We also present a framework that guides the implementation of our approach followed by description of our prototype application that uses educational multimedia metadata implemented in semantic web technologies.
\end{abstract}

\section{INTRODUCTION}

In recent years Mobile Learning has become very more popular, as a result of increased availability and significant improvement in the capabilities of mobile devices and network connectivity. Mobile learners have now access to rich educational multimedia content anytime and anywhere [1]. Learner have now control over what to learn when and where making it increasingly getting popular [2, 3]. Educational institutions and individuals around the world are now making their online learning videos available for free opening further avenues for learning while being on the move. Online educational multimedia, however, still present some challenges in the resource constrained mobile environment which need to be addressed.

The traditional one-size-fit-all approach towards learning resources is not suitable in the mobile learning paradigm as well, due to the diversity that exists in terms of mobile devices, network connectivity as well as learners' needs. Personalized learning resources can improve the efficiency of the learning process and help the learner take better advantage of the limited time and resource constrained device while on the move. Mobile devices have resource constraints in features like screen size, network characteristics, memory size and batterypower. There has been much research in adapting learning resources to the learner's needs and usage context [4] [5] [6]. The use of multimedia content specially poses many challenges in the delivery of mobile Learning context. Multimedia adaptation and personalization techniques have also been developed for Mobile Learning systems[3].

Traditionally, Content Adaptation and Universal Multimedia Access (UMA) [7] techniques have been used to adapt multimedia content based on the resource constraints of mobile devices. Limited Battery power is one big restricting factor in mobile learning. Online educational multimedia content when accessed over the mobile network quickly drains battery power as result of huge data transfer. Many techniques have been proposed to achieve mobile battery efficiency for multimedia applications. These techniques are not suitable for adapting learning multimedia as they do not consider the educational dimension in the adaptation process. Power Saving techniques must be integrated in Mobile Learning environments to enable learners in maximizing their learning experience.

Existing battery efficient multimedia adaptation techniques offer trade-off between user experience and battery life. These techniques tend to degrade quality in order to extend the battery life [8]. These methods lower encoding parameters like Frame per second, bitrates, colors and resolution. We in this paper, argue that efficacy of learning multimedia is dependent on the quality and modality. Lowering quality of multimedia beyond a certain level will always have a negative effect on learning outcome. We propose a novel power saving learning multimedia adaptation approach. This approach ensures maximum battery efficiency while keeping the educational multimedia effective. 
This paper is organized as follow. In Section II and III we discuss Adaptive Mobile Learning and Battery Efficient Multimedia adaptation. In Section IV we arrive at the conclusion that there is a need for power efficient educational multimedia adaptation. Section V introduces our proposed Content-Aware Power-Saving Educational Multimedia Adaptation (CAPS-EMA) approach. In Section VI we present a framework that enables the CAPS-EMA approach and finally in Section VII we briefly discuss our proof of concept implementation.

\section{ADAPTIVE MOBILE LEARNING}

Transforming multimedia learning resources in suitable versions in order to be efficiently delivered to meet the diverse needs of learners and mobile devices is a research area that is increasingly attracting a great deal of attention. Adaptive Mobile Learning uses content adaptation techniques to provide multiple personalized versions of the same learning content to tackle this challenge of diversity [9] [4].

Qing Tan in [5] Proposes a 5R adaptation framework, the aim of which is to provide a learning resource "at the right time, in the right location, through the right device, providing the right contents to the right learner". [10] discusses some issues arising due to the diversity that exists in learning on mobile devices. Over the years, many techniques have been proposed to provide adapted versions of learning resources based on user preferences, devices characteristics and user needs. Some of these techniques can be found in [6, 11-14].

Mobile Learning applications use existing generic multimedia adaptation techniques that are not developed specifically for educational multimedia. We, therefore, emphasize that multimedia adaptation techniques should be developed for educational multimedia that take into account the effects of adaptation on learning.

\section{POWER EFFICIENT MUlTimedia AdAPTATION}

Battery capacity has not advanced as much as other capabilities of mobile devices [15]. The power requirements of mobile devices are continuously increasing as mobile devices now come with increased processing capabilities, come with bigger screens and can communicate at higher data rates exposing the dire need for increased battery power efficiency.

Streaming of online Multimedia is one very power hungry application area. Considerable research has been done to improve battery efficiency while using online multimedia content. Multimedia adaptation techniques achieve power-saving by decreasing the media parameters like Frame per second, resolution, color, bitrates[16], [17] and changing even the modality of content. The general principle behind these approaches is to reduce the data size of multimedia which reduces the data transfer through wireless interface and results in usage of lesser battery power. A useful survey of Energy efficient techniques for multimedia applications can be found in [8].

McMullin et al. introduces a Power Save-based Adaptive Multimedia Delivery (PS-AMy) mechanism [18]. PS-AMy adapts multimedia streams in order to enable the streaming lasts longer. This system selects stream quality based on the remaining battery life and packet loss. Other non-application layer power saving approaches include multimedia network traffic shaping which uses Network Interface Card's Sleep and Awake modes[19] [20] [21] and screen's brightness [22] during playback.

We can see that existing generic adaptation methods do not consider the importance of visual information in different segments of the multimedia being adapted and any effects of adaptation on the information contained. Usefulness of multimedia for learning purposes may be lost after applying these methods and, therefore, are not suitable for Learning Multimedia.

We advocate that content adaptation techniques while deciding about the selected quality for delivery must consider the ultimate effect on the intended objective of the content. For example, while adapting learning multimedia, care must be taken that the quality is not degraded up to the extent that the multimedia becomes no longer useful for learning purpose, even though the quality may be good enough per user experience standards. On the other end, the quality of the content should not be high enough to achieve good userexperience; rather the lower quality should be selected based on the efficacy for learning purposes only. Quality of Experience in battery efficient mechanisms should be of secondary importance as against the efficacy of learning content.

\section{BATTERY EFFICIENCY IN MOBILE LEARNING}

Battery power constraint is a great challenge in taking maximum advantage of mobile devices for learning. Despite high dependency on battery power little has been done to improve battery efficiency in Mobile Learning Application. Moldovan et al in [23] emphasized that power saving techniques should be integrated in multimedia learning systems. Authosr have 
shown how multimedia encoding parameters effects battery life. The authors, however, focus on better user experience while recommending encoding parameters. The same authors in [24] suggest that significant battery power can be saved using their proposed content adaptation solution in Eco-Learn m-learning system. Subjective tests conducted on participants have shown that battery power saving through multimedia quality reduction does not significantly affect learning experience and the learning outcome. Their technique recommends learning multimedia in single uniform quality. Moreover, the learning contents used in experiments were from 20-30 seconds duration. We argue that the recommended quality may be suitable for this short segment but there is no evidence that the same quality will be suitable for entire educational multimedia, which may span up to an hour in most cases now a days.

Jalal et al in [25] have also proposed an approach of battery efficiency in mobile learning context. This work, however, is targeted at enabling the discovery of battery efficient version of an adaptive learning resource. In order to achieve this objective a metadata model for Adaptive Learning Resource has been proposed.

To the best our knowledge, no previous work has suggested any battery efficient adaptation mechanism for educational multimedia that provides optimum power saving while at the same time preventing any negative effect on learning as a result of adaptation.

\section{PROBLEMS WITH USING EXISTING POWER SAVING ADAPTATION TECHNIQUES FOR EDUCATIONAL MULTIMEDIA}

Based on the literature of adaptation methods and systems, we can conclude that there are significantly fewer Power-Save adaptation techniques at Application Level as compared to other types of power saving techniques. Furthermore, among application level power-save multimedia adaptation techniques, very few have considered educational multimedia.

1. First problem with existing generic techniques is that they do not have any mechanism to to consider the effect of quality degradation on the intended learning outcome. These methods are not content-aware. This means that quality degradation decisions are enforced irrespective of what the contents of multimedia are. This may make a multimedia content unhelpful for learning purpose if learning content was quality sensitive and required high quality only.
2. Second problem with these power-save adaptation techniques is that they select a single lower quality for the entire educational multimedia. Learning multimedia adapted to a single quality as a result either does not remain fully useful for learning or provides lesser than the possible power efficiency. The description of these issues is given below.

a. Battery efficiency at cost of compromising learning: Parts of an educational video content that contain quality sensitive visual information might require a higher quality than the selected one for successfully conveying information to make learning possible. Any lower quality selected through generic user-experience based methods cannot be safe in terms of learning for all segments of the same multimedia. Moreover, no generic method can decide about presentation qualities for all available online educational multimedia. Such systems would achieve power efficiency with unpredictable consequences for usefulness and may be damaging the educational value of resources.

b. Reduced Power efficiency: This problem has two dimensions. On one hand it may be possible that only few points the contents of multimedia require a higher quality. In order to meet the learning objectives, these few segments of media must be delivered in a higher quality. As the entire resource has to be delivered in a single quality to the mobile device, the entire content as result is delivered in a higher quality. This clearly results in spending more power than needed. As most of the resource could be delivered in a lower quality. A fragmented approached is clearly needed in this case. Only the fragments needing higher quality would be delivered in higher quality and the rest could be delivered in a lower quality saving battery power. This was the motivation beyond our proposed adaptation method, which adapts multimedia resource on segments by segments basis according to the needs and quality constraints.

It may also be possible that the selected lower quality - selected based on the acceptable user experience - may still be high enough for learning. The same content may be delivered safely in a lower quality version without compromising the learning process. A good example is a learning video where there is no significant visual 
information; the audio version in this case would provide the same learning opportunity. So the video version even if in a very lower quality would be a waste of battery power.

\section{CONTENT-AwARE POWER-SAVE EdUCATIONAL MULTIMEDIA ADAPTATION (CAPS-EMA)}

Based on the identified shorting comings in the existing techniques, we present a Content-Aware Power-Saving Educational Multimedia Adaptation (CAPS-EMA) mechanism. This mechanism will provide optimum battery efficiency while keeping the adapted content suitable for learning. The entire multimedia content is fragmented with multiple quality versions. Minimum Quality constraints are determined and placed on each fragment. If a fragment of multimedia has quality sensitive visual contents then it will be assigned a higher quality presentation constraint. On the other hand if another fragment of the video does not contain any important visual information at all and all the information is oral then the presentation constraint for such fragment will be audio quality. Most learning videos present visual information of different extents at different temporal point in the multimedia resource. Our proposed approach considers the effect of quality reduction on the information that is contained in each temporal fragment, by imposing quality constraints. This makes the delivery of each fragment in a different quality possible. Minimum acceptable quality varies from fragment to fragment. A fragment in our solution's context is a portion of multimedia segment in the temporal dimension that has same minimum presentation quality requirement to effectively achieve the learning aims. The output of our proposed approach is a fragmented, multi-quality and multimodal format. Figure 1 shows the difference between our approach and the traditional learning multimedia content adaptation approaches.

CAPS-EMA approach means that in order to achieve power saving the lower quality of multimedia is selected based on the requirement of each piece of content. This result in some segments of a multimedia to safely achieve its intended learning targets in a Low Quality, while the other part of the same multimedia resource will lose learning information in a Low Quality and must be delivered in a Medium Quality. In the same way, we can have some other part of the same resource that is delivered in Audio instead of even a very low quality video. This approach offers two fold benefits. On one hand it preserves the potential of adapted multimedia to remain effective for learning purposes. On the other hand it opens opportunities for even further power saving than the approaches based on quality of experience.

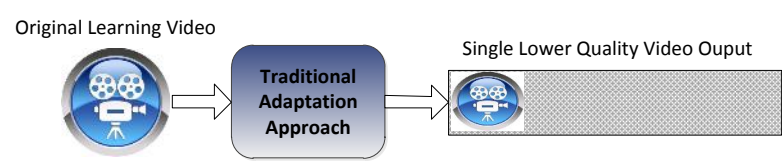

(a)- Traditional Adaptation Approach

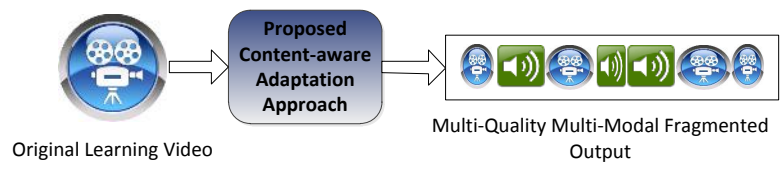

(b)- Proposed Content Aware Adaptation Approach

Figure 1: Comparison of Traditional and our Proposed Approaches

\section{CONCEPTUAL FRAMEWORK FOR CAPS- EMA}

The central concept of CAPS-EMA is fragmentation, appropriately versioned learning content and the associated metadata for presentation constraints. Multimedia learning content should be fragmented based on the minimum presentation quality requirements.

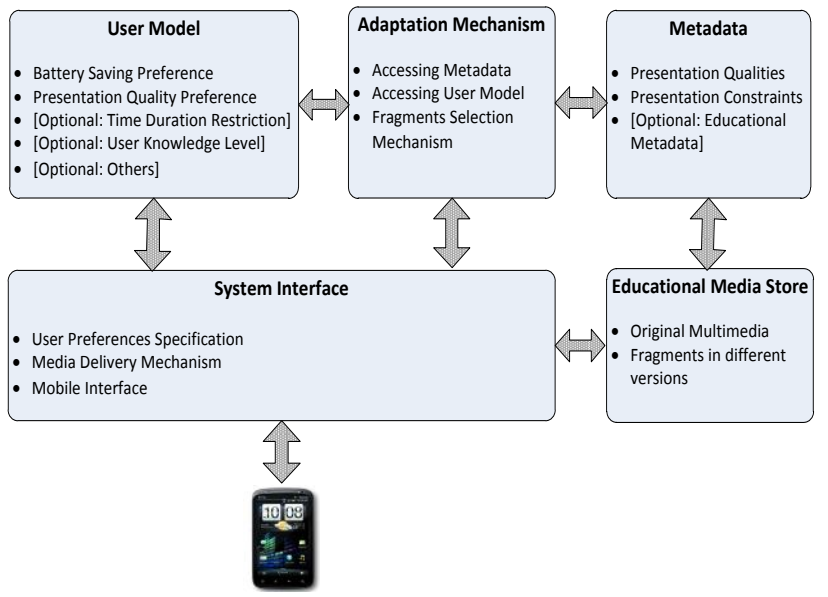

Figure 2: A conceptual Framework for Content-Aware PowerSave Educational Multimedia Adaptation

Multiple versions of the original content will enable the system to offer battery efficiency up to different extents, based on user preferences. The degree of power saving desired by mobile learner will determine the fragments to be selected and their individual quality. In case where learner desire maximum battery efficiency; in that case each fragment must be selected in the minimum most acceptable quality. The objective of metadata is to specify presentation quality information as well as 
minimum presentation quality constraints on each fragment.

Moreover, metadata can also include other information as needed for example, fragments' order number and their relative importance etc. The actual Adaptation process happens at the level of selection of the fragments and their versions. This selection is done on the basis of the user preferences (User Model) about the extent of desired power efficiency and the metadata. To take further advantage, user may be allowed to specify the knowledge level and the available time duration for learning. Every option in the user preference will have some role in the selection of fragments and their quality levels. We have developed a Fragmented EducationalMultimedia Model (FEMM) for useful metadata creation. FEMM is given below in Figure 3.

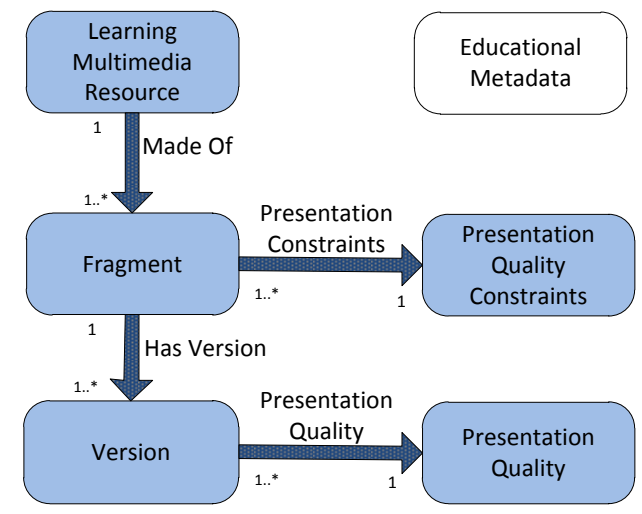

Figure 3: Abstract Fragmented Educational-Multimedia Model (FEMM)

\section{PROTOTYPE IMPLEMENTATION}

We have implemented the CAPS-EMA approach in a prototype system. We developed user interface in HTML5 and Mobile JQuery. We created metadata model in RDF. User preference was converted into SPARQL query for selecting of appropriate fragment versions. Some screen shots of the prototype system is shown in figure 4 . We used a sample learning video titled "Structure of Atom". We manually fragmented, created versions and entered the metadata. We specified Quality constraints after observing the content manually. In Figure 4, the top left screen shot shows user preference of no battery saving, in which case user will watch the original high quality video as one continuous fragment. In the next screenshot, user preference for maximum battery efficiency is selected using the sliderbar. This will result in each fragment being delivered in its minimum acceptable quality for learning. The bottom-left screenshot shows a fragment in lower quality. The next screenshot shows that one of the fragments is being played in audio quality. This audio quality is selected as constraint for this fragment mentions Audio as acceptable quality as there was no significant visual content in that fragment.

\section{CONCLUSION}

We proposed a novel Content Aware and Power Efficient approach of educational multimedia adaptation for mobile learning. The fundamental idea is that educational effectiveness of every segment of a learning multimedia should be considered in power-saving adaptation process. Our proposed approach adapts entire multimedia resource on fragment by fragment basis considering presentation constraints on each fragment, offering many advantages over existing adaptation techniques that deliver adapted multimedia in a single uniform quality. We ensure that maximum possible battery efficiency is achievable without negatively affecting the learning process. We present a conceptual framework and an abstract metadata model that guides the implementation of our power saving educational multimedia adaptation. We also briefly discuss our proof of concept prototype system.

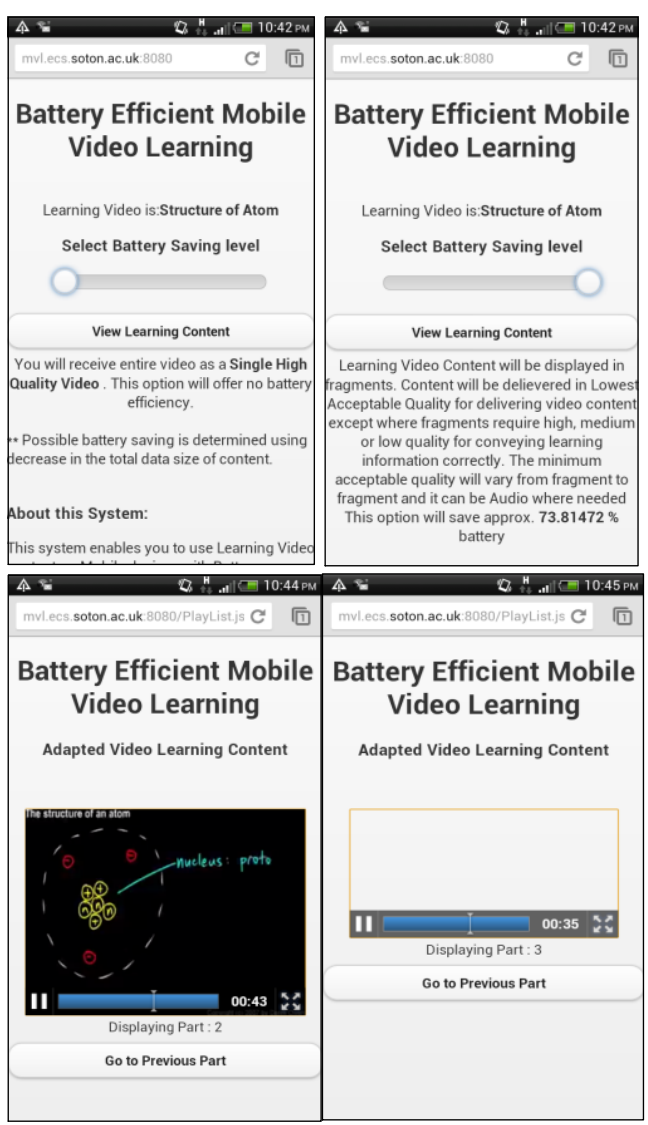

Figure 4: Mobile Screenshots of the prototype System 


\section{REFERENCES}

[1] Cheung, S.K., A study on the use of mobile devices for distance learning, in Hybrid Learning2012, Springer. p. 89-98.

[2] Bradley, C., et al., Design and development of multimedia learning objects for mobile phones. Mobile learning: Transforming the delivery of education and training, 2009: p. 157-182.

[3] Moldovan, A.-N. and C.H. Muntean. Towards personalised and adaptive multimedia in m-learning systems. in World Conference on E-Learning in Corporate, Government, Healthcare, and Higher Education. 2011.

[4] Sampson, D. and P. Zervas, Context-Aware Adaptive and Personalized Mobile Learning Systems, in Ubiquitous and Mobile Learning in the Digital Age, D.G. Sampson, et al., Editors. 2013, Springer New York. p. 3-17.

[5] Tan, Q., X. Zhang, and R.M.G. Kinshuk, The 5R Adaptation Framework for Location-Based Mobile Learning Systems, in 10th World Conference on Mobile and Contextual Learning2011: Beijing, China.

[6] Zhao, X., et al., Personalized Adaptive Content System for Context-Aware Mobile Learning. Int. J. of Computer Science and Network Security, 2008. 8(8): p. 153-161.

[7] Vetro, A., C. Christopoulos, and T. Ebrahimi, Universal multimedia access. IEEE Signal Processing Magazine, 2003. 20(2): p. 16.

[8] Hoque, M., M. Siekkinen, and J. Nurminen, Energy Efficient Multimedia Streaming to Mobile Devices-A Survey. 2012.

[9] Zervas, P., et al., Designing Tools for Context-Aware Mobile Educational Content Adaptation, in Ubiquitous and Mobile Learning in the Digital Age, D.G. Sampson, et al., Editors. 2013, Springer New York. p. 37-50.

[10] Zhao, X. and T. Okamoto, Adaptive multimedia content delivery for context-aware u-learning. International Journal of Mobile Learning and Organisation, 2011. 5(1): p. 46-63.

[11] Nguyen, V.A., V. Hanoi, and P. Van Cong. CAMLES: An adaptive mobile learning system to assist student in language learning. 2012. IEEE.

[12] García, A., et al. A system for adaptation of educational contents to learners and their mobile device. 2011. IEEE.

[13] Reveiu, A., I. Smeureanu, and M. Dardala. Content Adaptation in Mobile Multimedia System for M-Learning. 2008. IEEE.

[14] Yang, S.J.H., I.Y.L. Chen, and R. Chen. Applying content adaptation to mobile learning. 2007. IEEE.
[15] Adams, J. and G.M. Muntean. Power Save Adaptation Algorithm for Multimedia Streaming to Mobile Devices. in IEEE International Conference on Portable Information Devices (PORTABLE07). 2007.

[16] Moldovan, A.-N. and C.H. Muntean, Subjective Assessment of BitDetect-A Mechanism for Energy-Aware Multimedia Content Adaptation. Broadcasting, IEEE Transactions on, 2012. 58(3): p. 480-492.

[17] Trestian, R., et al. Energy consumption analysis of video streaming to android mobile devices. in Network Operations and Management Symposium (NOMS), 2012 IEEE. 2012. IEEE.

[18] McMullin, D., R. Trestian, and G.-M. Muntean, Power Savebased Adaptive Multimedia Delivery Mechanism, in The 9th Iformation Technology and Telecomunication Conference (IT\&T) 20092009

[19] Korhonen, J. and Y. Wang, Power-efficient streaming for mobile terminals, in Proceedings of the international workshop on Network and operating systems support for digital audio and video2005, ACM: Stevenson, Washington, USA. p. 39-44.

[20] Cornea, R., A. Nicolau, and N. Dutt, Content-aware Power Optimizations for Multimedia Streaming Over Wireless Networks, 2006, University of California.

[21] Anastasi, G., et al. A power-aware multimedia streaming protocol for mobile users. in International Conference on Pervasive Services, ICPS '05. 2005.

[22] Cornea, R., A. Nicolau, and N. Dutt, Using Annotations to Facilitate Power vs Quality Trade-offs in Streaming Applications, 2006: University of California.

[23] Moldovan, A.-N. and C.H. Muntean. Assessing Power Saving Techniques and Their Impact on E-learning Users. in 2009 IET China-Ireland International Conference on Information and Communications Technologies (CIICT 2009). 2009. National University of Ireland Maynooth.

[24] Moldovan, A., A. Molnar, and C. Muntean, EcoLearn: Battery power friendly e-learning environment for mobile device users. Learning-Oriented Technologies, Devices and NetworksInnovative Case Studies, 2011: p. 273-296.

[25] Asim Jalal, et al. Enabling the discovery of Adaptive Learning Resources for Mobile Learner. in 11th World Conference on Mobile and Contextual Learning: mLearn 2012. 2012. Helsinki, Finland. 